INTERNATIONAL DESIGN CONFERENCE - DESIGN 2018

https://doi.org/10.21278/idc.2018.0144

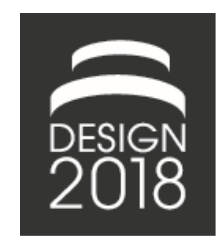

\title{
DEVELOPMENT OF A NEW SIMULATION-BASED PLATFORM FOR PRODUCT AND SERVICE CUSTOMER CO-DESIGN USING AI
}

\author{
Y. Feng, I. Kataoka, J. Yoshida and N. Nonaka
}

\begin{abstract}
This paper describes our new simulation-based platform for the customer co-design of products and service. This platform is based on the concept model that builds integrated digital models from the real world and analyzes from the simulation results. It explores and maximizes customer value using machine learning algorithms. The platform consists of a workflow constructor and a GUI builder with reusable nodes and templates. An example is given in the oil \& gas filed. The customer co-design GUI is built in 5 minutes and can be used to assess the business feasibility of the oil \& gas devices.
\end{abstract}

Keywords: co-design, simulation based design, artificial intelligence (AI), collaborative design, design tools

\section{Introduction}

One recent trend in the modern manufacturing industry is the transition from product-centred view of markets to a service-led model. Lusch and Vargo (2013) describe this transition as from goods-dominant (G-D) logic to service-dominant (S-D) logic. One of the most important rules in service-dominant logic is that value is not created by the manufacturers alone, but co-created with customers. In other words, value is mutually and equally created by the manufacturers and the customers during the interaction process (Lusch and Vargo, 2013).

Many companies have adopted the customer co-creation concept. For example, Hitachi, Ltd., one of the biggest manufacturers in Japan, has established a global centre for social innovation, which aims to deliver innovations by collaborating with customers and stakeholders (Funaki, 2017).

In the service-dominant logic, knowledge and skills are viewed as "operant resources" (Lusch and Vargo, 2013). It is pointed out that design is a very effective "operant resource" that can help companies in making attractive values through effective customer co-creation (Gemser, 2016). In this paper, we focus on the product and service design process in the manufacturing industry, especially in the B2B (Business to Business) industry.

In the manufacturing industry, computer simulation has long been an effective methodology for product design by shortening the design period and reducing prototyping cost. However, to the best of our knowledge, there are few digital aid tools for customer co-creation. One of such studies is in the KBE (Knowledge-Based Engineering) field. According to the review of Reddy et al. (2015), web-based approach towards KBE has gained much attention in the recent years. Web-based integrated product design platform has been developed for considering customer needs in the early phase of product development.

QFD (Quality Function Deployment) is another popular methodology that can be used to link the customer requirements with product design. In the QFD approach, customer requirements are linked to 
the products' design features, so that the designers can consider customer requirements in the early design phase. For example, Lin et al. (2006) develops a procedure to effectively link the customer requirements with design characteristics for product design. An analytic hierarchy process is adapted to evaluate the importance of customer requirements.

However, the above-mentioned research is only partially effective for customer co-design. In the webbased KBE systems, web is used as a media for visualizing design models and receiving customer requirements. Therefore, there is a time lag between customer desire and product design. Meanwhile, QFD is a tool for the company designers, making it difficult for realizing customer co-design in which feedback from customers is a key feature.

There were also studies that apply QFD matrices to product customization by combining them with knowledge-based systems (Kutschenreiter-Praszkiewicz, 2013). Wang and Chen (2012) tried to use QFD for collaborative product design by adopting fuzzy algorithms and linear integer programming. There were also many studies focusing on processing customer review data for better product and system design (Kim, 2015). It is worth mentioning that most of those studies focused on the B2C (Business to Consumer) business models and assumed end-users as "customers".

Recently, the advances in the digital technologies, especially in the fields of AI (Artificial Intelligence), IoT (Internet of Things), cloud computing and big data, have had a profound impact on manufacturing. One of the most important influences is that customer-related data, as well as other various data, are being integrated into "big data" and are being utilized in the optimization of the lifecycle of products and services (Tao et al., 2018).

In this study, we take advantage of the state-of-the-art digital technologies and apply them in developing an integrated customer co-design platform. Our aim is to develop a digital environment that is optimized for customer co-design, especially for the B2B business. It uses computer simulation to evaluate the performance of a product or service. It builds customized GUI that is suitable for customer co-design. It also uses AI to create design plans that satisfy the customer's requirement.

\section{Customer co-design platform using simulation and AI}

In this chapter, we describe the concept, system architecture and contents of our proposed customer codesign platform.

\subsection{Concept}

Figure 1 describes the concept of the proposed simulation-based customer co-design platform. It consists of three layers, i.e., the physical layer, the cyber layer, and the value layer. The physical layer contains data of the products, systems, and services that exist in the real world. It also includes public data in all media. The cyber layer is a mapping of the physical layer. It includes simulation of the products, systems, and services. It also includes models based on hypotheses and models of social phenomena. The value layer is the ultimate goal of the customers, which includes KPIs (Key Performance Indices) such as safety, profit, assurance ... etc.

Our goal is to maximize customer value in an efficient way. To realize this goal, we have categorized three methodologies, i.e., synchronization, integration, and customization.

The first methodology is "synchronization", which connects the physical layer and the cyber layer. It uses sensor and/or experimental data of the physical world to increase the speed and accuracy of simulation. It is a high-speed, high-precision mapping of the physical world to the cyber space. The key technology we adopted in synchronization is the data assimilation (Evensen, 2009). Data assimilation has its application mainly in weather prediction and it is considered as a frontier branch of atmospheric and oceanic sciences (Miyoshi et al., 2014). Recently, some researchers began to expand its usage to industrial simulation. For example, Kusano et al. (2016) have used data assimilation to estimate the uncertain parameters in the engine room of a hydraulic excavator.

The second methodology is "integration", which is an integrated cross-domain modelling and simulation technology in the cyber layer. To predict the outcome of a system, it is necessary to simulate the behaviour of all the key products and services within the system. Traditional simulation is too timeconsuming to achieve this goal. We have developed a multi-fidelity total integrated simulation 
technology, which allows the users to freely control the fidelity of each component model. For example, Oh et al. (2017) have used the technology on a high pressure pump system.

The third methodology is "customization", which links the cyber layer and the value layer. With the help of a modern supercomputer, the cyber layer is able to produce a huge database of simulation results. Optimization is necessary to explore the simulation database and obtain best solutions for the customers. However, traditional optimization technology such as GA (Genetic Algorithm) requires tremendous number of simulations and is usually time-consuming, making it unsuitable for interactive co-design. To solve this problem, we adopted DNN (Deep Neural Network) which is a fast machine learning algorithm (Hinton and Salakhutdinov, 2006) to explore the simulation results database and predict models that maximize customer value.

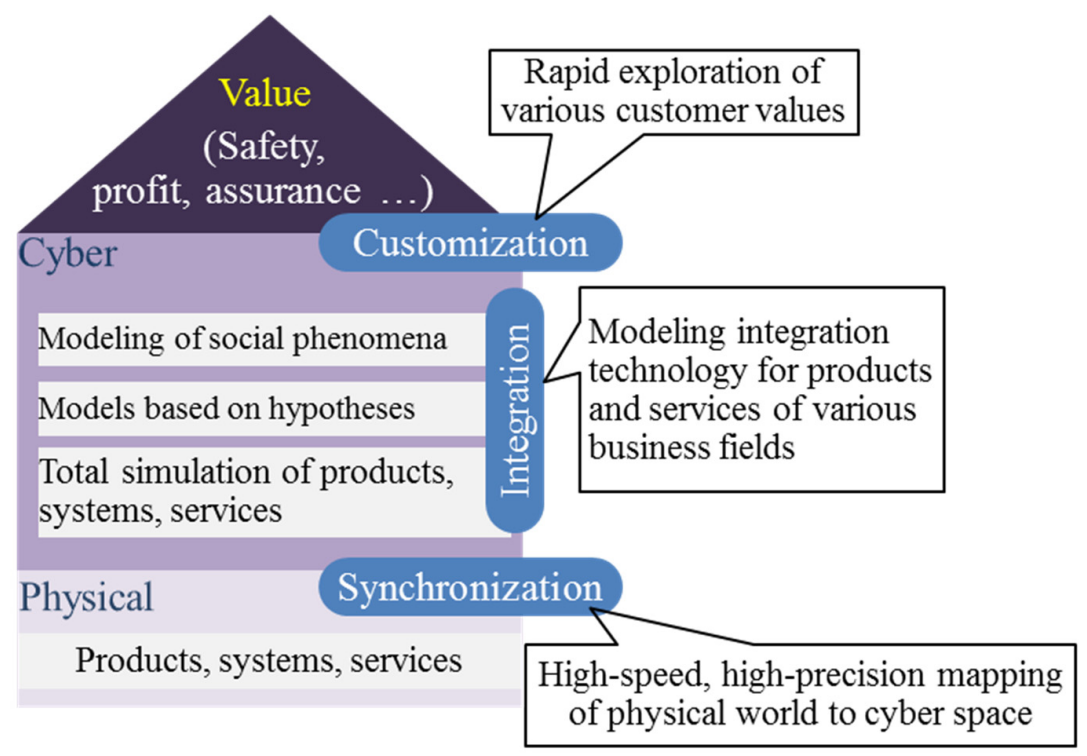

Figure 1. Conceptual model of the customer co-design platform

We are developing a methodology to apply DNN to product/service design problems (Kataoka et al., 2016). In the traditional method, design plans are obtained as a result of "forward problem". The designer uses design tools to calculate the performance of candidate design plans and compare the performance with customer specification. It is usually a trial-and-error process until the customer specification is satisfied. In the proposed method, we tackle the design problem as a "reverse problem". We first construct a database of specifications and design plans. Then we use the database as training data for the DNN model and construct the relationship between specifications and design plans. We then use DNN again to predict the design plan once customer specification is received. In this way, the design plans can be shown to the user in a very short time, making it possible for the customer to give feedback to the designer on the spot. We call this process "interactive customer co-creation".

\subsection{System architecture}

The system architecture of the customer co-design platform is shown in Figure 2. It is based on serverclient system. The client machine contains workflow constructor which defines calculation procedure, and a dashboard builder for constructing the GUI. The GUI is linked to the workflow and displays the results of the workflow calculation. The server machine contains programs that manage the workflow and corresponding database. The calculation procedure is defined in the client machine and executed in the server machine. For time-consuming programs such as heavy simulation and machine learning, there is an option to execute them in a supercomputer other than the server machine. The calculation results are saved as database in the server side and can be visualized during customer co-creation. This system architecture enables the users to use the system anywhere as well as provide data security. 


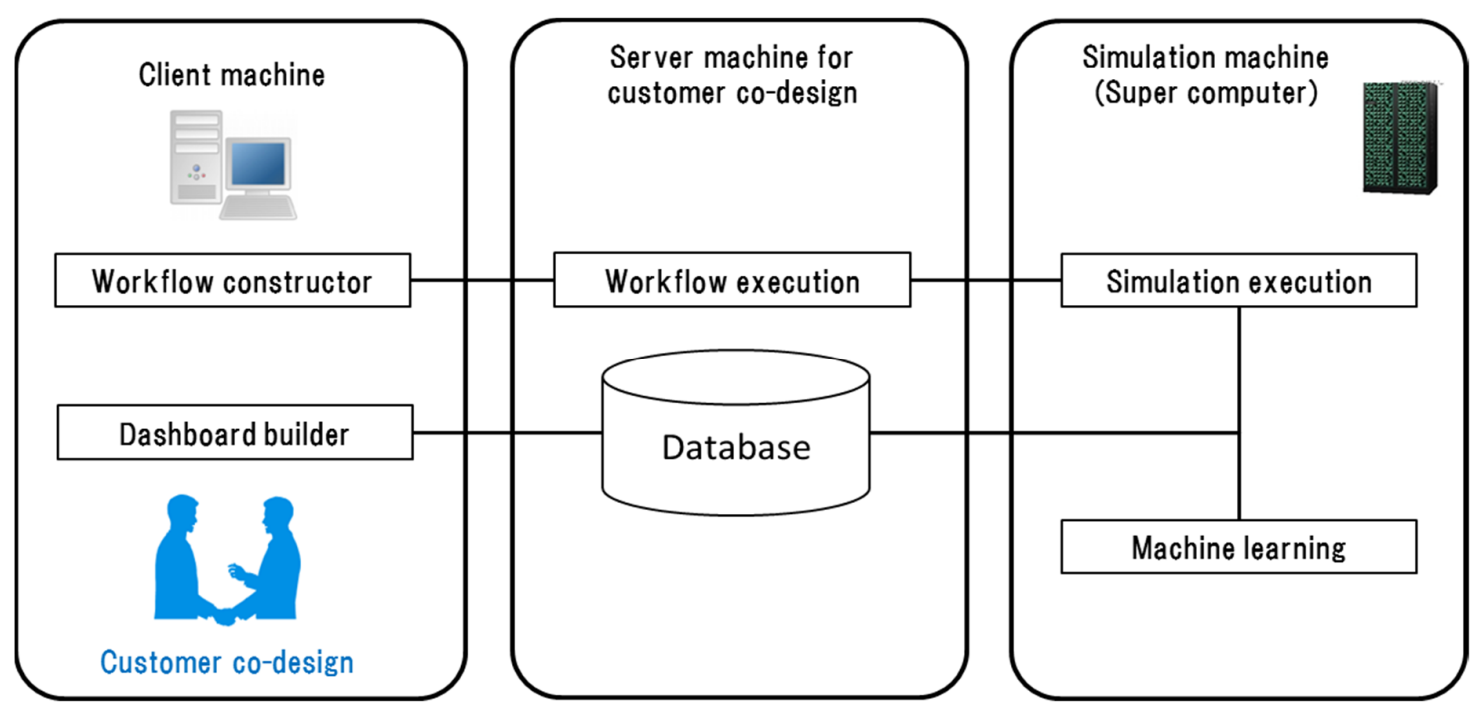

Figure 2. System architecture of customer co-design platform

\subsection{Workflow constructor}

The workflow constructor is used to build customized calculation procedures and execute the calculation automatically. Construction of calculation procedures is usually a tedious and time consuming work. In this research, we have built a workflow constructor that can easily perform parameter survey or construct machine learning procedures. It is based on the KNIME open-source analysis software developed at the University of Konstanz.

As shown in Figure 3, analysis models or programs are defined as nodes. These nodes are registered to KNIME and have a common interface and thus can be easily reused. Users can build customized workflows by simply drag \& drop the predefined nodes and connect them. The defined workflows are then saved in the server. Furthermore, we have prepared templates for the frequently used workflows such as parameter survey or machine learning, making it even easier to build new workflows.

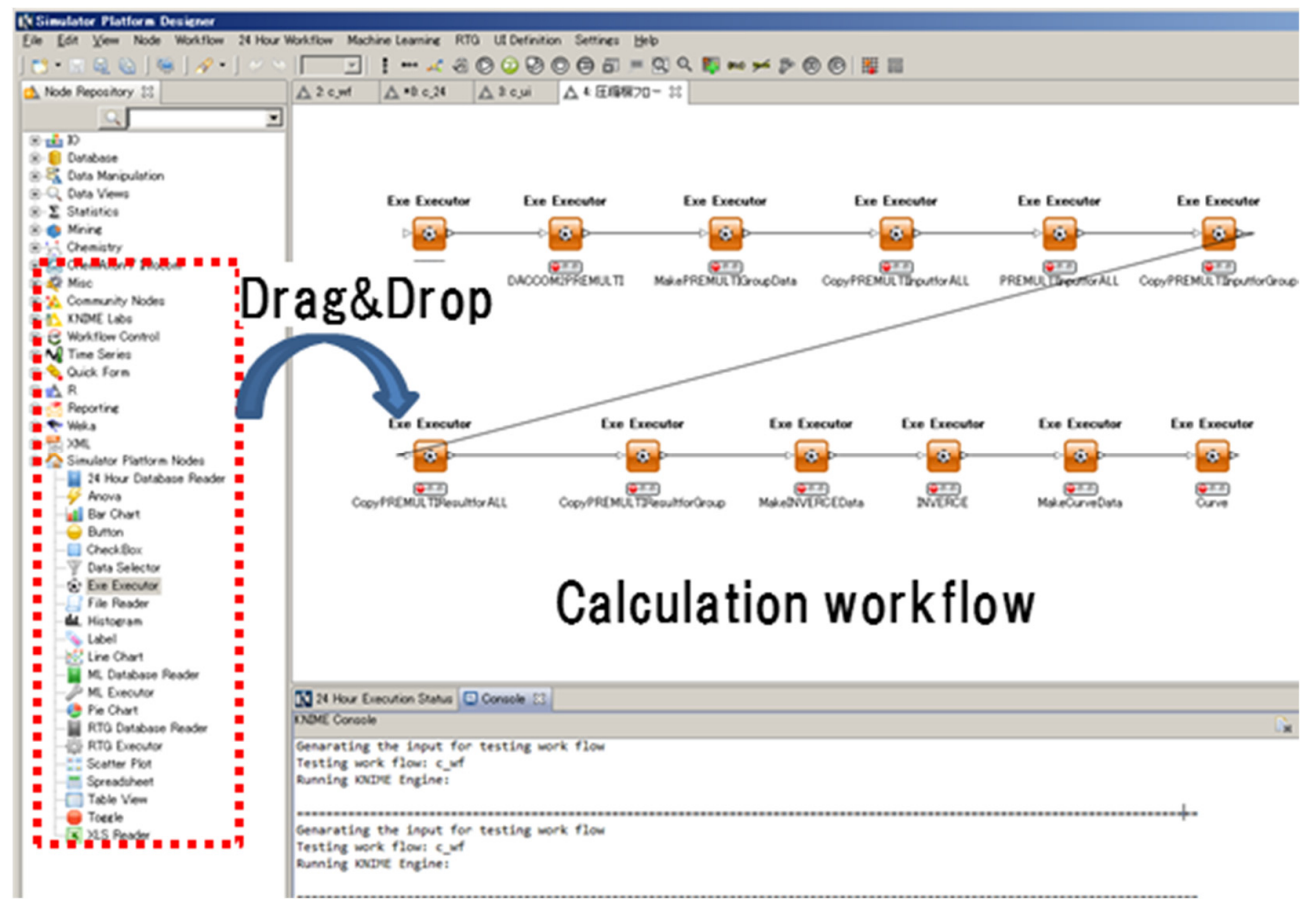

Figure 3. Image of building calculation workflow from predefined nodes 


\subsection{Dashboard style GUI builder}

GUI plays an important role in the customer co-design process. We have developed a dashboard builder that can display the customer input, design plan and simulation results in one screen. Users can easily customize the style and layout of the customer co-design GUI. To achieve this, we have predefined visualization nodes that are commonly used in GUI design. By dragging \& dropping the nodes, it is easy to build a GUI that fits the customer's flavour. An image of the dashboard style GUI builder is shown in Figure 4.

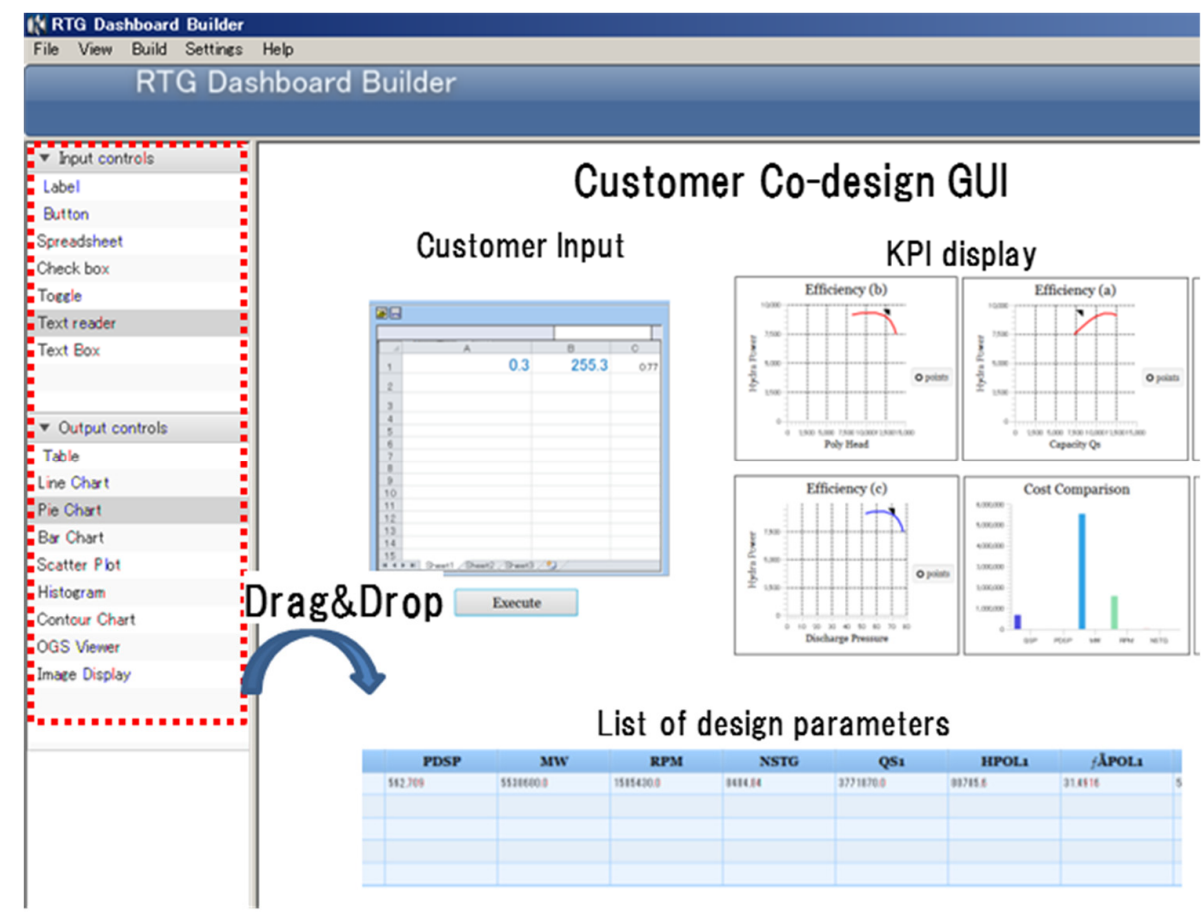

Figure 4. System architecture of customer co-design platform

Customer co-design GUI has three main components: the input area, the execution area, and the output area. The input area is where customers can input their requirements. The execution area will trigger the execution of simulation workflows, which uses the customer requirements as an input. The execution area is linked with the simulation workflows. The output area displays the simulation results, design plans, KPIs, and/or list of design parameters.

The built customer co-design GUI is a standalone application that can be executed at any client machine. As described in Section 2.1, design plans are predicted by using DNN and can be delivered to the customer almost real-time. Therefore, the GUI application enables the interactive value co-creation between the customers and the designers.

\subsection{Use cases of the proposed platform}

There are two types of potential users for our proposed platform. The first one is designers who develop product or service systems. The design process essentially translates the customer needs into design parameters, which typically requires numerous trial-and-errors. The workflow constructor can aid the designers to organize the design tools into intuitive workflows. The dashboard builder can help designers to build a graphically rich environment in which designers can perform the trial-and-errors more efficiently.

The second one is the sales engineers or sale persons who interact with the customers directly. Sale engineers or sales persons are typically unfamiliar with the design tools and have less knowledge about the design parameters. Some of them are not familiar with engineering tools such as the design constructor or the dashboard builder. A typical use case is that the sale engineers or sale persons will 
collaborate with the designers. The designers will create the workflows and build the dashboard GUI. The sales persons can then use the dashboard as a demonstration tool to appeal to the customers. Since the customers in the B2B business are well familiar with the products/services, the dashboard can serve as a common environment for the sales person and the customer to explore product/service features and scenarios together, which is exactly the essence of customer co-creation.

\section{Application}

We apply the customer co-design platform to oil \& gas business feasibility tool. In this application, the targeted customer is NOC (National Oil Company) or IOC (International Oil Company). The main purpose is to design oil \& gas devices such as ESPs (Electrical Submersible Pumps) that are used to boost the production of oil/gas. The design of the apparatus depends largely on the properties of the reservoir, the layout of the wellbores... etc. Since those data are fully owned by the customers, it is necessary to co-design the system with customers. Therefore, we build a tool based on the customer codesign platform to evaluate the performance of the devices and the feasibility of those devices in the oil\&gas business.

\subsection{Simulation model}

Reservoir simulations are usually based on FEM (Finite Element Method) and are usually timeconsuming. In this research, we have developed a simplified simulation model based on the network method (Bruce, 1943). The network method uses the analogy between the fluid flows in the reservoir to the electric flow in a circuit network.

Figure 5 illustrates the input and output of the oil \& gas simulator with the ability to evaluate the effect of ESPs. The input of the simulator is geometry data of the reservoir, calculation conditions, properties of the ESP device, and parameter data of the reservoir, wellbore and fluid. The output of the simulator is the pressure distribution of the reservoir, as well as the oil/gas production rate.
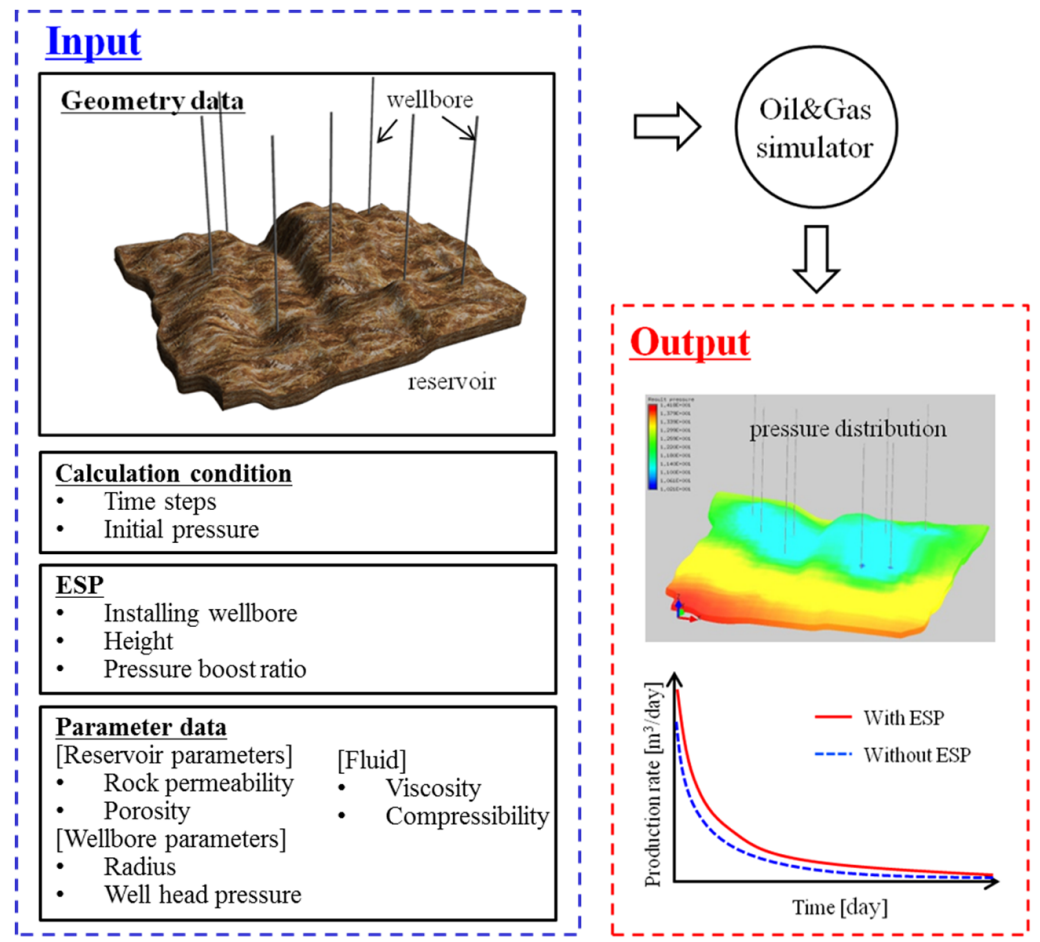

Figure 5. Input and output of the oil \& gas simulator

Besides the network-model-based reservoir simulator, we also developed a device simulator that calculated the effect of ESP devices. To evaluate the economic benefit of the devices, we developed a cost simulator which calculates the economic KPIs of the oil/gas field. The economic KPIs include 
CAPEX (Capital Expenditure), OPEX (Operational Expenditure), NPV (Net Present Value), net profit ... etc. Table 1 shows a list of the simulators used in the oil\&gas customer co-creation system.

Table 1. List of simulators used in the oil\&gas customer co-creation system

\begin{tabular}{|l|l|l|}
\hline \multicolumn{1}{|c|}{ Simulator } & \multicolumn{1}{|c|}{ Input } & \multicolumn{1}{c|}{ Output } \\
\hline Reservoir simulator & $\begin{array}{l}\text { Reservoir geometry, fluid properties, } \\
\text { wellbore parameters }\end{array}$ & $\begin{array}{l}\text { Pressure distribution, } \\
\text { production rate }\end{array}$ \\
\hline Device simulator & $\begin{array}{l}\text { Device specification, operation } \\
\text { condition }\end{array}$ & $\begin{array}{l}\text { Pressure change, energy } \\
\text { consumption }\end{array}$ \\
\hline Cost simulator & Oil/gas price, & Economic KPIs \\
\hline
\end{tabular}

\subsection{Workflow and GUI construction}

Having developed the simulators, we also developed an in-house GUI application for obtaining customer inputs. The GUI application contains a reservoir and multiple wellbores. It allows the users to set the number and position of the wellbores, as well as editing the properties of the reservoir and oil/gas properties. Oil/gas prices can also be set in the GUI application.

Figure 6 shows the workflow and GUI of the customer co-design system for oil\&gas. Using the Microsoft Active X technology, we are able to embed the GUI application into the dashboard system. Other dashboard components are built from the visualization nodes. The dashboard components are linked to the corresponding workflows or database in the server. The construction time for the oil\&gas customer co-design system is about 5 minutes. Without using the platform, it will typically take several days for a designer to develop such a system.

Using the built customer co-design system, sales engineers can interact with the NOC or IOC customers to explore business feasibility by configuring the layout of the wellbores and adjusting the position of the ESP. Simulator will then be executed in the cloud and the results will be fetched to the local PC or tablet. KPIs will immediately be calculated and displayed in the dashboard. The NOC or IOC customers can then judge the usefulness of the ESP devices by looking at the KPIs. The customers can also adjust the input parameters and execute the workflow repeatedly to optimize the scenario. Therefore, this tool can be used for the efficient co-design of ESP between oil/gas companies and the ESP developers.

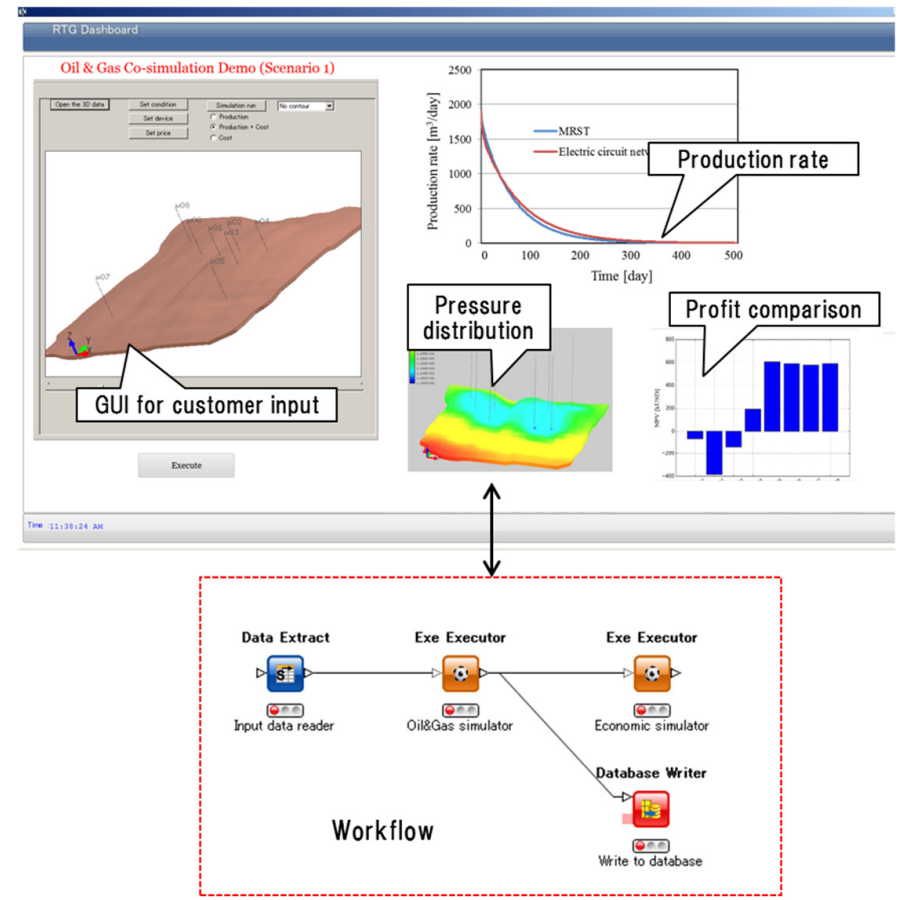

Figure 6. Workflow and GUI of oil\&gas customer co-design 


\section{Conclusion}

This paper gives an overview of our new simulation-based platform for the customer co-design of products and service. This platform is based on the concept model that builds integrated digital models from the real world and construct digital database from the simulation results. It explores and maximizes customer value using machine learning algorithms. The platform consists of a workflow constructor and a GUI builder. Customized calculation workflows and dashboard GUIs can be built easily from predefined nodes. The platform is mainly for the $\mathrm{B} 2 \mathrm{~B}$ business and it has two types of potential users, one is the product/service designer, the other is the sales engineers or sale persons. An example is given in the oil \& gas filed. The customer co-design GUI is built in 5 minutes and can be used to assess the business feasibility of the oil \& gas devices.

\section{References}

Bruce, W.A. (1943), "An electrical device for analysing oil-reservoir behaviour", Transactions of the AIME, Vol. 151 No. 1, pp. 112-124. https://doi.org/10.2118/943112-G

Evensen, G. (2009), Data Assimilation, Springer, Berlin, Heidelberg. https://doi.org/10.1007/978-3-642-03711-5

Funaki, K. (2017), "Customer co-creation to deliver innovation in the digital era", Hitachi Review, Vol. 66, No. 6, pp. 38-40.

Gemser, G. (2016), "Co-creating the future: design roles, practices and tools for effective customer co-creation", 11th EAD Conference Proceedings: The Value of Design Research. https://doi.org/10.7190/ead/2015/58

Hinton, G.E. and Salakhutdinov, R.R. (2006), "Reducing the dimensionality of data with neural networks", Science, Vol. 313 No. 5786, pp. 504-507. https://doi.org/10.1126/science.1127647

Kataoka, I., Zhang, Z. and Nonaka, N. (2016), "Development of machine learning technology for product design space", Proceedings of the 12th Optimization Symposium, Sapporo, Japan, December 6- 7, 2016, JSME Design Division. https://doi.org/10.1299/jsmeoptis.2016.12.1211

Kim, K. (2015), "Understanding and utilizing requirements and needs for product and system design", Journal of Integrated Design and Process Science, Vol. 19 No. 2, pp. 1-3. https://doi.org/10.3233/jid-2015-0013

Kusano, K., Yamakawa, H. and Ikeda, K. (2016), "Estimation of Uncertain Parameters for Thermal and Fluid Analysis in Engine Room of Construction Machinery Using Data Assimilation", Proceedings of the ASME 2016 Fluids Engineering Division Summer Meeting, Washington, DC, USA, July 10 - 14, 2016, ASME Fluids Engineering Division, pp. V01AT03A020. https://doi.org/10.1115/FEDSM2016-7898

Kutschenreiter-Praszkiewicz, I. (2013), "Product configuration using knowledge based system", Journal of Machine Engineering, Vol. 13 No. 2, pp. 26-35.

Lin, M.C., Wang, C.C. and Chen, T.C. (2006), "A strategy for managing customer-oriented product design", Concurrent Engineering, Vol. 14, No. 3 pp. 231-244. https://doi.org/10.1177/1063293X06068390

Miyoshi, T., Kondo, K. and Imamura, T. (2014), "The 10,240-member ensemble Kalman filtering with an intermediate AGCM", Geographical Research Letters, Vol. 41 No. 14, pp. 5264-5271. https://doi.org/10.1002/2014GL060863

Oh, U., Kusano, K., Nonaka, N. and Yamakawa, H. (2017), "Multi-fidelity total integrated simulation technology for high pressure pump with squeeze film effect", SAE International Journal of Passenger Cars - Mechanical Systems, Vol. 10 No. 2, pp. 507-513. https://doi.org/10.4271/2017-01-1325

Tao, F., Cheng, J., Qi, Q., Zhang, M., Zhang, H. and Sui, F. (2018), "Digital twin-driven product design, manufacturing and service with big data", The International Journal of Advanced Manufacturing Technology, Vol. 94 No. 9-12, pp. 3563-3576. https://doi.org/10.1007/s00170-017-0233-1

Wang, C. and Chen, J. (2012), "Using quality function deployment for collaborative product design and optimal selection of module mix", Computer \& Industrial Engineering, Vol. 63 No. 4, pp. 1030-1037. https://doi.org/10.1016/j.cie.2012.06.014

Dr. Yixiang Feng, Senior Researcher

Hitachi, Ltd., Research and Development Group

832-2 Horiguchi, Hitachinaka, Ibaraki, 3120034 Hitachinaka, Japan

Email: yixiang.feng.cq@hitachi.com 